\title{
Association between physical activity and conversion from mild cognitive impairment to dementia
}

\author{
Yeo Jin Kim', Kyung-Do Han², Min Seok Baek ${ }^{3}$, Hanna Cho ${ }^{3^{*}}$ (D) Eun Joo Lee ${ }^{4}$ and Chul Hyoung Lyoo ${ }^{3}$
}

\begin{abstract}
Background: Physical activity has been suggested to prevent the conversion of mild cognitive impairment (MCl) to dementia in patients. We investigated the association between the continuance and regularity of physical activity and the risk of developing dementia in patients with $\mathrm{MCl}$.

Methods: We analyzed 6-year followed up data for 247,149 individuals in the National Health Insurance Service (NHIS) cohort of Korea who were enrolled between January 1, 2009, and December 31, 2015. The patients were divided into four groups: those who did not engage in physical activity consistently (Never-PA group), those who initiated physical activity (Initiation-PA group), those who ceased physical activity (Withdrawal-PA group), and those who performed physical activity consistently (Maintenance-PA group). We also divided the patients into two groups: those who engaged in physical activity irregularly (Irregular-PA) and those who undertook physical activity regularly (Regular-PA).

Results: When the risk for the Never-PA group was set as the benchmark (ref $=1)$, the Maintenance-PA group had the lowest incidence of dementia of the Alzheimer type (DAT) compared to the other groups $(\mathrm{HR}=0.82,95 \% \mathrm{Cl}$ 0.79-0.86). The DAT risk of the Initiation-PA group ( $H R=0.89,95 \% \mathrm{Cl} 0.85-0.93)$ was lower than the Never-PA group. In addition, compared to the Irregular-PA group, the Regular-PA group had a 15\% reduced risk for developing DAT.

Conclusions: Although no causal inference could be made, continued regular physical activity in patients with $\mathrm{MCl}$ is associated with a protective effect against developing DAT. Moreover, ceasing physical activity could halt this protective effect.
\end{abstract}

Keywords: Mild cognitive impairment, Physical activity, Continuance, Regularity

\section{Background}

Mild cognitive impairment (MCI) is associated with a high risk of progression to dementia [1-5]. There are currently no approved disease-modifying treatments, and lifestyle modifications have become an important strategy to prevent its progression. Physical activity is considered to be the most important interventional

\footnotetext{
*Correspondence: iguhanna@naver.com; iguhanna@yuhs.ac

${ }^{3}$ Department of Neurology, Gangnam Severance Hospital, Yonsei University College of Medicine, 211, Eonju-ro, Gangnam-gu, Seoul 06273, Republic of Korea

Full list of author information is available at the end of the article
}

strategy for the prevention of dementia, with previous studies showing that physical activity reduces the likelihood of disease progression in early dementia [6].

The types, intensity, duration, and frequency of physical activity have been shown to impact the protective effect of physical activity [7]. Leisure-time physical activity is associated with a reduced risk of dementia, while work-related physical activity has not shown an association with a protective effect [8]. High-intensity physical activity was found to be more effective than lowintensity physical activity [9], while the duration and

C C The Author(s). 2020 Open Access This article is licensed under a Creative Commons Attribution 4.0 International License, which permits use, sharing, adaptation, distribution and reproduction in any medium or format, as long as you give appropriate credit to the original author(s) and the source, provide a link to the Creative Commons licence, and indicate if changes were made. The images or other third party material in this article are included in the article's Creative Commons licence, unless indicated otherwise in a credit line to the material. If material is not included in the article's Creative Commons licence and your intended use is not permitted by statutory regulation or exceeds the permitted use, you will need to obtain permission directly from the copyright holder. To view a copy of this licence, visit http://creativecommons.org/licenses/by/4.0/. The Creative Commons Public Domain Dedication waiver (http://creativecommons.org/publicdomain/zero/1.0/) applies to the data made available in this article, unless otherwise stated in a credit line to the data. 
frequency of physical activity is also an important factor that can reduce the risk of cognitive decline. In a recent meta-analysis, physical activity of more than 16 weeks was associated with a greater improvement in cognition [10]. In addition, the incidence rate of dementia was lower in subjects who engaged in physical activity more than twice per week $[11,12]$. However, the association between withdrawal or initiation of physical activity and prevention against cognitive decline remains poorly understood. Similarly, the importance of the regularity of physical activity needs further investigation. We thus sought to investigate the effect of physical activity continuance and regularity on the rate of conversion from MCI to dementia of the Alzheimer type (DAT) using longitudinal data from the National Health Insurance Service (NHIS) covering Korean patients that were followed up for 6 years.

\section{Methods}

\section{Data source and study population}

The present study was conducted using data from the national health insurance claims database established by the National Health Insurance Service (NHIS) of Korea [13]. The NHIS registration is mandatory for all Koreans, except for those with low incomes. People with low incomes are enrolled in the Medical Aid program; however, since 2006, these patient data have also been incorporated into the NHIS database. Therefore, the information obtained from the NHIS database represents health information for almost all patients in Korea [14].

The NHIS database includes demographic information, medical use, disease information, lifestyle habits, and basic laboratory data [15]. We defined diagnoses using the International Classification of Disease, Tenth Revision, Clinical Modification (ICD-10-CM) codes. The general health examination is administered to all citizens over 20 years every 2 years. Data from all patients with an MCI diagnosis from January 1, 2009, to December 31,2015 , were included $(n=975,030)$. Of these MCI patients, we selected only those who had undertaken a health examination within 2 years after their MCI diagnosis $(n=424,684)$. We excluded patients aged $<40$ years $(n=4643)$ and only selected those with matched examination data within 2 years before their MCI diagnosis $(n=261,814)$. Patients with dementia diagnosed before the index date were excluded $(n=14,665)$. The index date of this study was second health examination date. In the end, 247,149 individuals were included in this study (Fig. 1). The period from 1st health examination to MCI diagnosis was 12.0 months and the period from MCI diagnosis to 2nd health examination was 10.8 months (Supplementary Table 1).

\section{Standard protocol approvals, registrations, and patient consent}

We obtained written informed consent from each patient. This study was approved by the Institutional Review Board of Gangnam Severance Hospital, and all methods were performed in accordance with the approved guidelines and regulations.

\section{Definition of $\mathrm{MCl}$ and DAT}

$\mathrm{MCI}$ was diagnosed using the ICD-10-CM codes and includes F067. The codes for DAT were F00 and G30.

\section{Physical activity measurements}

Data on physical activity were collected via self-report questionnaires. The extent of physical activity was assessed using the Korean version of the international physical activity questionnaire (K-IPAQ) short form, focusing on physical activity performed in the last 7 days. The 7 items of the IPAQ identify the total minutes over the last 7 days spent on moderate- and vigorous-intensity physical activity. Participants were asked how many days per week they spent for each activity over $10 \mathrm{~min}$ and how many minutes per activity they spent per day over the last 7 days. Vigorous activities included carrying heavy objects, running, aerobics, and fast biking. Moderate activities included carrying light items, bike riding at normal speed, and doubles tennis.

\section{Classification of $\mathrm{MCl}$ patients}

We classified patients with MCI into four groups (Fig. 2). The "Never-PA" group included those patients who did not engage in physical activity before or after MCI diagnosis. We defined the physical activity to be vigorous or moderate physical activity over 10 min more than 1 days per week. The "Initiation-PA" group included patients who did not engage in physical activity before the diagnosis of MCI but initiated physical activity after the diagnosis of MCI. The "Withdrawal-PA" group included patients who engaged in physical activity before the diagnosis of MCI but stopped physical activity after the diagnosis of MCI. The "Maintenance-PA" group included patients who engaged in physical activity before and after MCI diagnosis. We also classified patients with MCI into two groups according to the regularity of physical activity. The "Regular-PA" group included patients who performed physical activity regularly, while the "Irregular-PA" group included patients who engaged in physical activity irregularly. We defined regular physical activity to be vigorous physical activity more than 3 days per week or moderate physical activity more than 5 days per week. 
$\mathrm{N}=975,030$

Subjects who had a mild cognitive impairment

diagnosis in 2009-2015

4,643 subjects younger than 40-year-old were excluded

$\mathrm{N}=261,814$

Subjects who had matched health examination data within two years before $\mathrm{MCI}$ diagnosis

14,665 subjects were excluded with diagnosis of dementia before the index year

$\mathrm{N}=247,149$

Subjects eligible for inclusion

Fig. 1 Flow chart of the study population

\section{Statistical analysis}

The data from the NHIS database are presented as mean values \pm standard deviation (SD) for continuous variables and percentages for categorical variables. Differences between groups were confirmed using analysis of variance (ANOVA) for continuous variables and chi-square test for categorical variables. Cox proportional hazard models were used to assess the risk of dementia. Dementia risk was expressed as the hazard ratio (HR) with $95 \%$ confidence interval $(95 \% \mathrm{CI})$. Incidence rates were calculated per 1000 person-years. $P$ values were two-tailed, and statistical significance was considered at $P<0.05$. All statistical analyses were performed using SAS V.9.3.

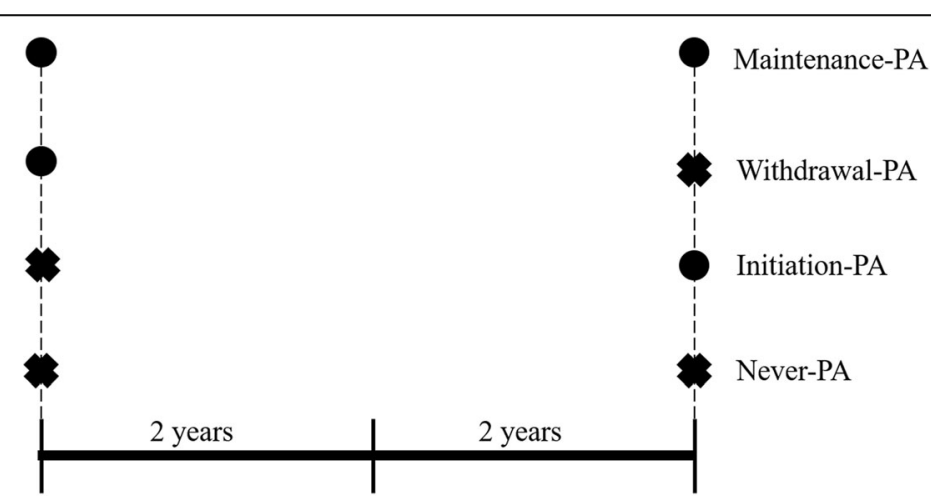

Health examination

MCI diagnosis 2009.1.1 2015.12.31

Health examination

Fig. 2 Classification of the four groups of $\mathrm{MCl}$ patients. The circle indicated that the patient had performed moderate to vigorous physical activity in the last 7 days. The cross indicated that the patient had not performed physical activity in the last 7 days 


\section{Results}

\section{Demographics and baseline characteristics}

Detailed demographic and clinical characteristics of the participants are presented in Table 1 . The mean age of the patients in the Never-PA group was highest, while the mean age of the patients in the Maintenance-PA group was lowest. The Never-PA group had the highest proportion of females. In addition, the Never-PA group had the highest proportion of patients with hypertension or diabetes mellitus. Compared to the Irregular-PA group, the Regular-PA group is younger and had the higher proportion of females. The Irregular-PA group had the higher proportion of patients with hypertension (Supplementary Table 2).

Risk of dementia according to continuity of physical activity after diagnosis of mild cognitive impairment Of the 247,149 patients analyzed, 23,015 patients converted to dementia. Of these 23,015, 17,733 (77\%) were diagnosed with DAT.

Table 2 shows the risk of DAT according to the continuity of physical activity. The Maintenance-PA group showed the lowest risk of dementia. Compared to the Never-PA group, the risk of DAT was reduced in the Initiation-PA group and Maintenance-PA group (for the Initiation-PA group: HR 0.89, 95\% CI 0.85-0.93; for the Maintenance-PA group: HR 0.82, 95\% CI 0.79-0.86), while the risk of DAT was the same between the NeverPA group and Withdrawal-PA group (for WithdrawalPA group: HR 1.00, 95\% CI 0.96-1.04). There were no interactions between the comorbidity and the continuity of physical activity (Supplementary Table 3). The association between initiating physical activity and dementia risk in females was greater than in males, while the association between continuance of physical activity and dementia risk in males was greater (in females for the Initiation-PA group: HR 0.88, 95\% CI 0.84-0.92; for Withdrawal-PA group: HR 0.99, 95\% CI 0.94-1.03; for Maintenance-PA group: HR 0.82 95\% CI 0.78-0.86; in males for the Initiation-PA group: HR 0.92, 95\% CI 0.86-0.97; for Withdrawal-PA group: HR $0.98,95 \% \mathrm{CI}$ 0.92-1.04; for Maintenance-PA group: HR $0.7795 \% \mathrm{CI}$ 0.73-0.82). However, these gender-specific differences were not statistically significant.

\section{Risk of dementia according to regularity of physical activity}

Table 3 shows DAT risk according to the regularity of physical activity. When the risk for the Irregular-PA group was set as a reference $(r e f=1)$, compared to the Irregular-PA group, the Regular-PA group had a $15 \%$ reduced risk of Alzheimer's disease after adjusting for age, gender, and vascular risk factors (HR 0.85, 95\% CI $0.81-0.88)$.

\section{Discussion}

Our nationwide population-based longitudinal cohort study analysis shows that continued physical activity in patients with MCI is associated with a lower risk of DAT. It appears that the decision to start physical

Table 1 Baseline characteristics of the study population

\begin{tabular}{|c|c|c|c|c|c|}
\hline & $\begin{array}{l}\text { Never-PA } \\
(n=99,873)\end{array}$ & $\begin{array}{l}\text { Initiation-PA } \\
(n=45,598)\end{array}$ & $\begin{array}{l}\text { Withdrawal-PA } \\
(n=45,014)\end{array}$ & $\begin{array}{l}\text { Maintenance-PA } \\
(n=56,664)\end{array}$ & $P$ value \\
\hline Age, year & $68.7 \pm 9.3$ & $66.4 \pm 9.2$ & $67.2 \pm 9.1$ & $64.6 \pm 9.1$ & $<0.0001$ \\
\hline Female, $n(\%)$ & $72,836(72.9)$ & $30,862(67.7)$ & $30,131(66.9)$ & $32,204(56.8)$ & $<0.0001$ \\
\hline Hypertension, $n(\%)$ & $58,178(58.3)$ & $24,777(54.3)$ & $24,889(55.3)$ & $28,962(51.1)$ & $<0.0001$ \\
\hline Diabetes mellitus, $n(\%)$ & $22,448(22.5)$ & $9542(20.9)$ & $10,062(22.4)$ & $11,171(19.7)$ & $<0.0001$ \\
\hline Dyslipidemia, n (\%) & $45,981(46.0)$ & $20,976(46.0)$ & $20,893(46.4)$ & $25,596(45.2)$ & 0.001 \\
\hline Obesity*,$n(\%)$ & $37,096(37.1)$ & $16,617(36.4)$ & $16,407(36.5)$ & $20,160(35.6)$ & $<0.0001$ \\
\hline Body mass index, $\mathrm{kg} / \mathrm{m}^{2}$ & $24.16 \pm 3.25$ & $24.15 \pm 3.05$ & $24.15 \pm 3.10$ & $24.13 \pm 2.88$ & 0.257 \\
\hline Smoking, $n(\%)$ & & & & & $<0.0001$ \\
\hline Non-smoker & $82,345(82.5)$ & $36,071(79.1)$ & $36,017(80.0)$ & $40,576(71.6)$ & \\
\hline Ex-smoker & $9812(9.8)$ & $6093(13.4)$ & $5665(12.6)$ & $11,689(20.6)$ & \\
\hline Current smoker & $7716(7.7)$ & $3434(7.5)$ & $3332(7.4)$ & $4399(7.8)$ & \\
\hline Drinking, $n(\%)$ & & & & & $<0.0001$ \\
\hline Non-drinker & $84,755(84.9)$ & $35,743(78.4)$ & $36,858(81.9)$ & $39,453(69.6)$ & \\
\hline Mild to moderate drinker & $12,598(12.6)$ & $8593(18.9)$ & $6953(15.5)$ & $15,241(26.9)$ & \\
\hline Heavy drinker & $2520(2.5)$ & $1262(2.8)$ & $1203(2.7)$ & $1970(3.5)$ & \\
\hline Time to conversion from $\mathrm{MCl}$ to dementia (months) & $31.3 \pm 16.6$ & $31.6 \pm 16.7$ & $31.6 \pm 16.8$ & $31.0 \pm 16.4$ & $<0.0001$ \\
\hline
\end{tabular}

PA physical activity

*Body mass index $\geq 25 \mathrm{~kg} / \mathrm{m}^{2}$ 
Table 2 Risk of dementia according to continuity of physical activity

\begin{tabular}{lllll}
\hline & $\begin{array}{l}\text { Never-PA } \\
(\boldsymbol{n}=\mathbf{9 9 , 8 7 3 )}\end{array}$ & $\begin{array}{l}\text { Initiation-PA } \\
(\boldsymbol{n}=\mathbf{4 5 , 5 9 8 )}\end{array}$ & $\begin{array}{l}\text { Withdrawal-PA } \\
(\boldsymbol{n}=\mathbf{4 5 , 0 1 4 )}\end{array}$ & $\begin{array}{l}\text { Maintenance-PA } \\
(\boldsymbol{n}=\mathbf{5 6 , 6 6 4 )}\end{array}$ \\
\hline DAT cases, $n$ (\%) & $8658(8.7 \%)$ & $2888(6.3 \%)$ & $3445(7.7 \%)$ & $2742(4.8 \%)$ \\
$\begin{array}{l}\text { DAT incidence (per 1000 person-years) } \\
\text { Unadjusted HR (95\% Cl) }\end{array}$ & 33.25 & 24.11 & 29.13 & 18.77 \\
DAT & 1 (ref) & $0.73(0.70-0.76)$ & $0.88(0.84-0.91)$ & $0.56(0.54-0.59)$ \\
Adjusted HR* $(95 \% \mathrm{Cl})$ & & & & $0.82(0.79-0.86)$ \\
DAT & 1 (ref) & $0.89(0.85-0.93)$ & $1.00(0.96-1.04)$ & \\
\hline
\end{tabular}

$H R$ hazard ratio, $\mathrm{Cl}$ confidence interval, DAT dementia of the Alzheimer type, PA physical activity

*Adjusted for age, gender, hypertension, diabetes mellitus, dyslipidemia, body mass index, history of smoking, and history of alcohol intake

activity leads to a lower risk of DAT, while ceasing physical activity may cause the risk of DAT to increase again. In addition, a higher frequency of physical activity appears to prevent conversion from MCI to DAT (moderate-intensity physical activity more than 5 days per week or vigorous-intensity physical activity more than 3 days per week).

We observed that the Maintenance-PA group had 18\% fewer dementia conversions than the Never-PA group, while the Initiation-PA group had $11 \%$ less dementia conversion than the Never-PA group. We interpret this to indicate that continuing physical activity occurring at both time points was more effective than initiating a new physical activity between the two time points. Evidence suggests that the longer the duration of physical activity, the greater the effect of physical activity on cognitive function [16]. The findings for the Initiation-PA group may therefore reflect the shorter duration of physical activity compared to the Maintenance-PA group.

There are at least two major mechanisms by which continuous physical activity may prevent the conversion from mild cognitive impairment to dementia. Physical activity increases the expression of neurotrophic factors such as brain-derived neurotrophic factor (BDNF), insulin-like growth factor 1 (IGF-1), and vascular endothelial growth factor (VFGF). BDNF is important for maintaining neuronal development and for exercise-

Table 3 Risk of dementia according to regularity of physical activity

\begin{tabular}{lll}
\hline & Irregular-PA & Regular-PA* \\
\hline Unadjusted HR $(95 \% \mathrm{Cl})$ & & \\
$\begin{array}{l}\text { Dementia of the Alzheimer type } \\
\text { Adjusted HR }(95 \% \mathrm{Cl})^{\dagger}\end{array}$ & 1 (ref) & $0.70(0.68-0.73)$ \\
Dementia of the Alzheimer type & 1 (ref) & $0.85(0.81-0.88)$ \\
\hline
\end{tabular}

$H R$ hazard ratio, $\mathrm{Cl}$ confidence interval, $P A$ physical activity *Vigorous-intensity physical activity $\geq 3$ days per week or moderate-intensity physical activity $\geq 5$ days per week

${ }^{\dagger}$ Adjusted for age, gender, hypertension, diabetes mellitus, dyslipidemia, body mass index, history of smoking, and history of alcohol intake related improvements in cognitive function. IGF-1 and VFGF play important roles in neurogenesis and angiogenesis and influence the induction of hippocampal BDNF. Animal and clinical studies have demonstrated that physical activity can increase neurotrophic factors such as BDNF and IGF-1. After physical activity, the release of BDNF from the brain is enhanced [17, 18], with 6 months of resistance exercise sufficient to increase serum levels of IGF-1 in older adults [19].

Physical activity also increases cerebral blood flow (CBF). After 12 weeks of physical activity, CBF has been shown to increase in the anterior cingulate cortex [20] and hippocampal CBF increased in elderly patients with subjective memory complaints after 16 weeks of physical activity [21]. CBF is thought to maintain cerebral perfusion to help maintain brain volume. Previous studies have shown that physical activity is associated with increased regional gray and white matter volume including areas such as the hippocampus, and prefrontal and cingulate cortices [22, 23].

Ceasing regular physical activity appears to halt the positive effects on the body that the physical activity was eliciting. One small study found that elderly subjects who undertook endurance training for more than 15 years had reduced CBF in specific brain regions including the hippocampus after stopping physical activity [24]. Stopping physical activity also reduces cardiorespiratory fitness and muscle mass and increases glucose intolerance which might adversely affect cognitive function [25-27]. These adverse effects associated with stopping physical activity arise within 10-20 days. Our findings also showed that the dementia conversion rate was lower in the Initiation-PA group than the Withdrawal-PA group, so the duration of the positive effects of physical activity does not appear to be very long.

We also investigated the association between regularity of physical activity and dementia risk. The Regular-PA group showed a $15 \%$ reduction in dementia conversion compared to the Irregular-PA group. However, not all studies have shown that physical activity improves cognitive function, and the effects of exercise on MCI 
have shown inconsistencies. In a review of 14 randomized controlled trials, the majority had insufficient evidence of the benefits of physical activity on cognitive function in patients with MCI [28], whereas another review showed that aerobic exercise elicited beneficial effects on cognitive function in MCI patients [29]. A possible explanation for these discrepancies is that each study involved different conditions including the type, intensity, frequency, and duration of physical activity. There have also been three large multidomain trials, FINGER [30], MAPT [31], and PreDIVA [32], which sought to investigate whether lifestyle modifications prevent cognitive impairment. Among these studies, only the FINGER study showed positive results, which could be because it involved active physical training that was different to the PreDIVA and MAPT studies.

Effects of physical activity on cognitive function in demented patients were also unclear. A randomized control study reported that moderate- to high-intensity exercise training could not slow cognitive impairment in patients with mild to moderate dementia [33]. Many of studies included in a meta-analysis of randomized control trials showed beneficial effects of physical activity on cognitive function in patients with dementia. However, because of unclear methodology, it was difficult for these studies to conclude that the effect of physical activity on dementia patients was proved. On the other hand, several relatively large studies included in the meta-analysis reported no effects on global cognition [34].

We note several limitations to our study. First, the evaluation of physical activity was based on selfreporting by the participants and could be open to bias. Second, when we analyzed the association frequency of physical activity with dementia, we could not control for disease severity due to a lack of cognitive function information. However, since the subjects were diagnosed with mild cognitive impairment, the level of baseline cognition would presumably not be very different. Third, the participants' physical activity characteristics, such as type, intensity, duration, and frequency, were free to change during the study period, but we did not take into account the effect of such changes. Moreover, we only measured the presence or absence of physical activity at two distinct time points. Fourth, the biomarker information could not be used for diagnosis. From the claim data used in this study, only clinical diagnosis based on ICD-10 could be obtained. Therefore, some of patients with dementia of the Alzheimer type in this study might be non-Alzheimer's disease patients. Fifth, because of the data access policy, it was not possible to use data for people not included in the study, so it was impossible to perform additional analyses on people who were not included in the inclusion criteria, such as those who had health examination only once or who did not be diagnosed MCI. In addition, we did not investigate the relation between physical activity and incident MCI. Sixth, although this study had a longitudinal design, it was difficult to have causal inference. Since this was an observational study, possible confounder might cause the effect. Lastly, as the cohort included participants of Korean ethnicity, caution should be taken if generalizing these findings to other populations. However, our study involved a very large sample size of over 247,000 adults. In addition, we focused on the continuity and regularity of physical activity, representing more specific factors that can reduce the risk of DAT.

\section{Conclusion}

Our study showed that continued physical activity in patients with MCI is associated with a lower risk of DAT. It appears that the decision to start physical activity leads to a lower risk of DAT, while ceasing physical activities may cause the risk of DAT to increase again. In addition, regular physical activity appears to prevent conversion from MCI to DAT. However, further research is needed into the duration of benefit from physical activity and the potential biological mechanisms involved. In addition, prospective studies are needed to clarify the result of this study.

\section{Supplementary information}

Supplementary information accompanies this paper at https://doi.org/10. 1186/s13195-020-00707-1.

Additional file 1: Supplementary Tables. Supplementary Table 1. Pre- and post-MCl diagnosis period in each group. Supplementary Table 2. Baseline characteristics of the study population between Irregular and Regular-PA. Supplementary Table 3. Interactions of comorbidities with continuity of physical activity.

\section{Abbreviations}

MCl: Mild cognitive impairment; DAT: Dementia of the Alzheimer type; NHIS: (Korean) National Health Insurance Service; ICD-10-CM: International Classification of Disease, Tenth Revision, Clinical Modification; K-IPAQ: Korean version of the international physical activity questionnaire; PA: Physical activity; SD: Standard deviation; ANOVA: Analysis of variance; HR: Hazard ratio; 95\% Cl: 95\% confidence interval; BDNF: Brain-derived neurotrophic factor; IGF-1: Insulin-like growth factor 1; VFGF: Vascular endothelial growth factor; CBF: Cerebral blood flow; FINGER: The Finnish Geriatric Intervention Study to Prevent Cognitive Impairment and Disability; MAPT: The Multidomain Alzheimer Preventive Trial; PreDIVA: The Prevention of Dementia by Intensive Vascular Care

\section{Acknowledgements}

Not applicable

Standard protocol approvals, registrations, and patient consent We obtained written informed consent from each patient. This study was approved by the Institutional Review Board of Gangnam Severance Hospital, and all methods were performed in accordance with the approved guidelines and regulations.

Authors' contributions

Yeo Jin Kim interpreted the data and drafted the manuscript. Kyung-Do Han contributed to the study design and data analysis. Min Seok Baek 
contributed to the study conceptualization and the discussion of results. Hanna Cho contributed to the study design, data interpretation, and revision of the manuscript. Eun Joo Lee contributed to the data analysis. Chul Hyoung Lyoo contributed to the study design and data interpretation. The authors read and approved the final manuscript.

\section{Funding}

This research was supported by faculty research grant of Yonsei University College of Medicine (6-2018-0068), a grant from the 2020 Research Grant of Gangnam Severance Hospital Research Committee, Basic Science Research Program through the National Research Foundation of Korea (NRF) funded by the Ministry of Education (NRF2020R1F1A1076154 \& NRF 2018R1D1A1B07049386), and a grant of the Korea Health Technology R\&D Project through the Korea Health Industry Development Institute (KHIDI) funded by the Ministry of Health \& Welfare, Republic of Korea (Grant number: HI18C1159), and the National Research Foundation of Korea grant funded by the Korean government (NRF-2017R1C1B2011637 and NRF2019R1H1A1035599).

\section{Availability of data and materials}

The original anonymized data used in this analysis was obtained from NHIS of Korea. The dataset from NHIS is not publicly available due to restricted access. However, any researcher requiring access to the data can obtain it directly through a license agreement, including the payment of appropriate license fees.

\section{Ethics approval and consent to participate}

The study was approved by the institutional review board of Gangnam Severance Hospital.

\section{Consent for publication}

Not applicable

\section{Competing interests}

The authors declare that they have no competing interests.

\section{Author details}

'Department of Neurology, Chuncheon Sacred Heart Hospital, Hallym University College of Medicine, Chuncheon, Republic of Korea. ${ }^{2}$ Department of Biostatics, College of Medicine, The Catholic University of Korea, Seoul, Republic of Korea. ${ }^{3}$ Department of Neurology, Gangnam Severance Hospital, Yonsei University College of Medicine, 211, Eonju-ro, Gangnam-gu, Seoul 06273, Republic of Korea. ${ }^{4}$ Big Data Steering Department, National Health Insurance Service, Wonju, Republic of Korea.

\section{Received: 13 February 2020 Accepted: 15 October 2020}

Published online: 11 November 2020

\section{References}

1. Petersen RC, Aisen P, Boeve BF, Geda YE, Ivnik RJ, Knopman DS, et al. Mild cognitive impairment due to Alzheimer disease in the community. Ann Neurol. 2013;74(2):199-208

2. Prestia A, Caroli A, Van Der Flier WM, Ossenkoppele R, Van Berckel B, Barkhof $\mathrm{F}$, et al. Prediction of dementia in $\mathrm{MCl}$ patients based on core diagnostic markers for Alzheimer disease. Neurology. 2013;80(11):1048-56.

3. Vos SJ, Verhey F, Frölich L, Kornhuber J, Wiltfang J, Maier W, et al. Prevalence and prognosis of Alzheimer's disease at the mild cognitive impairment stage. Brain. 2015;138(5):1327-38.

4. Wolk DA, Price JC, Saxton JA, Snitz BE, James JA, Lopez OL, et al. Amyloid imaging in mild cognitive impairment subtypes. Ann Neurol. 2009;65(5): 557-68. https://doi.org/10.1002/ana.21598.

5. Han JW, Kim TH, Lee SB, Park JH, Lee JJ, Huh Y, et al. Predictive validity and diagnostic stability of mild cognitive impairment subtypes. Alzheimer's Dementia. 2012;8(6):553-9. https://doi.org/10.1016/j.jalz.2011.08.007.

6. Burns JM, Cronk BB, Anderson HS, Donnelly JE, Thomas GP, Harsha A, et al. Cardiorespiratory fitness and brain atrophy in early Alzheimer disease. Neurology. 2008;71(3):210-6. https://doi.org/10.1212/01.wnl.0000317094 86209.cb.

7. Stephen R, Hongisto K, Solomon A, Lonnroos E. Physical activity and Alzheimer's disease: a systematic review. J Gerontol A Biol Sci Med Sci. 2017; 72(6):733-9. https://doi.org/10.1093/gerona/glw251.
8. Rovio S, Kareholt I, Viitanen M, Winblad B, Tuomilehto J, Soininen H, et al. Work-related physical activity and the risk of dementia and Alzheimer's disease. Int J Geriatr Psychiatry. 2007;22(9):874-82. https://doi.org/10.1002/ gps. 1755.

9. Sofi F, Valecchi D, Bacci D, Abbate R, Gensini GF, Casini A, et al. Physical activity and risk of cognitive decline: a meta-analysis of prospective studies. J Intern Med. 2011;269(1):107-17. https://doi.org/10.1111/j.1365-2796.2010. 02281.x.

10. Jia RX, Liang $J H, X u Y$, Wang YQ. Effects of physical activity and exercise on the cognitive function of patients with Alzheimer disease: a meta-analysis. BMC Geriatr. 2019;19(1):181. https://doi.org/10.1186/s12877-019-1175-2.

11. Larson EB, Wang L, Bowen JD, McCormick WC, Teri L, Crane P, et al. Exercise is associated with reduced risk for incident dementia among persons 65 years of age and older. Ann Internal Med. 2006;144(2):73. https://doi.org/10. 7326/0003-4819-144-2-200601170-00004.

12. Rovio S, Kåreholt I, Helkala E-L, Viitanen M, Winblad B, Tuomilehto J, et al. Leisure-time physical activity at midlife and the risk of dementia and Alzheimer's disease. Lancet Neurol. 2005;4(11):705-11. https://doi.org/10. 1016/s1474-4422(05)70198-8.

13. Song SO, Jung CH, Song YD, Park CY, Kwon HS, Cha BS, et al. Background and data configuration process of a nationwide population-based study using the Korean national health insurance system. Diabetes Metab J. 2014; 38(5):395-403. https://doi.org/10.4093/dmj.2014.38.5.395.

14. Lee SR, Choi EK, Han KD, Cha MJ, Oh S. Trends in the incidence and prevalence of atrial fibrillation and estimated thromboembolic risk using the CHA2DS2-VASC score in the entire Korean population. Int J Cardiol. 2017; 236:226-31. https://doi.org/10.1016/j.ijcard.2017.02.039.

15. Choi JB, Lee EJ, Han KD, Hong SH, Ha US. Estimating the impact of body mass index on bladder cancer risk: stratification by smoking status. Sci Rep. 2018:8(1):947. https://doi.org/10.1038/s41598-018-19531-7.

16. Cabral DF, Rice J, Morris TP, Rundek T, Pascual-Leone A, Gomes-Osman J. Exercise for brain health: an investigation into the underlying mechanisms guided by dose. Neurotherapeutics. 2019;16(3):580-99. https://doi.org/10. 1007/s13311-019-00749-w.

17. Seifert T, Brassard P, Wissenberg M, Rasmussen P, Nordby P, Stallknecht B, et al. Endurance training enhances BDNF release from the human brain. Am J Physiol Regul Integr Comp Physiol. 2010;298(2):R372-7. https://doi.org/10. 1152/ajpregu.00525.2009.

18. Kurdi FN, Flora R. The impact of physical exercise on brain-derived neurotrophic factor (BDNF) level in elderly population. Open Access Maced J Med Sci. 2019;7(10):1618-20. https://doi.org/10.3889/oamjms.2019.337.

19. Cassilhas RC, Viana VA, Grassmann V, Santos RT, Santos RF, Tufik S, et al. The impact of resistance exercise on the cognitive function of the elderly. Med Sci Sports Exerc. 2007;39(8):1401-7. https://doi.org/10.1249/mss Ob013e318060111f.

20. Chapman SB, Aslan S, Spence JS, Defina LF, Keebler MW, Didehbani N, et al. Shorter term aerobic exercise improves brain, cognition, and cardiovascular fitness in aging. Front Aging Neurosci. 2013;5:75. https://doi.org/10.3389/ fnagi.2013.00075

21. Burdette $J H$, Laurienti PJ, Espeland MA, Morgan A, Telesford Q, Vechlekar $C D$, et al. Using network science to evaluate exercise-associated brain changes in older adults. Front Aging Neurosci. 2010;2:23. https://doi.org/10. 3389/fnagi.2010.00023.

22. Ruscheweyh R, Willemer C, Kruger K, Duning T, Warnecke T, Sommer J, et al. Physical activity and memory functions: an interventional study. Neurobiol Aging. 2011;32(7):1304-19. https://doi.org/10.1016/..neurobiolaging.2009.08.001.

23. Kleemeyer MM, Kuhn S, Prindle J, Bodammer NC, Brechtel L, Garthe A, et al. Changes in fitness are associated with changes in hippocampal microstructure and hippocampal volume among older adults. Neuroimage. 2016;131:155-61. https://doi.org/10.1016/j.neuroimage.2015. 11.026 .

24. Alfini AJ, Weiss LR, Leitner BP, Smith TJ, Hagberg JM, Smith JC. Hippocampal and cerebral blood flow after exercise cessation in master athletes. Front Aging Neurosci. 2016;8:184. https://doi.org/10.3389/fnagi.2016.00184.

25. McGuire DK, Levine BD, Williamson JW, Snell PG, Blomqvist CG, Saltin B, et al. A 30-year follow-up of the Dallas Bed Rest and Training study. Circulation. 2001;104(12):1358-66. https://doi.org/10.1161/hc3701.096099.

26. Klausen K, Andersen LB, Pelle I. Adaptive changes in work capacity, skeletal muscle capillarization and enzyme levels during training and detraining. Acta Physiol Scand. 1981;113(1):9-16. https://doi.org/10.1111/j.1748-1716. 1981.tb06854.x. 
27. Rogers MA, King DS, Hagberg JM, Ehsani AA, Holloszy JO. Effect of 10 days of physical inactivity on glucose tolerance in master athletes. J Appl Physiol (1985). 1990;68(5):1833-7. https://doi.org/10.1152/jappl.1990.68.5.1833.

28. Gates N, Fiatarone Singh MA, Sachdev PS, Valenzuela M. The effect of exercise training on cognitive function in older adults with mild cognitive impairment: a meta-analysis of randomized controlled trials. Am J Geriatr Psychiatry. 2013;21(11):1086-97. https://doi.org/10.1016/j.jagp.2013.02.018.

29. Zheng G, Xia R, Zhou W, Tao J, Chen L. Aerobic exercise ameliorates cognitive function in older adults with mild cognitive impairment: a systematic review and meta-analysis of randomised controlled trials. Br J Sports Med. 2016;50(23):1443-50. https://doi.org/10.1136/bjsports-2015095699

30. Ngandu T, Lehtisalo J, Solomon A, Levälahti E, Ahtiluoto S, Antikainen R, et al. A 2 year multidomain intervention of diet, exercise, cognitive training, and vascular risk monitoring versus control to prevent cognitive decline in at-risk elderly people (FINGER): a randomised controlled trial. Lancet. 2015; 385(9984):2255-63. https://doi.org/10.1016/s0140-6736(15)60461-5.

31. van Charante EPM, Richard E, Eurelings LS, van Dalen J-W, Ligthart SA, van Bussel EF, et al. Effectiveness of a 6-year multidomain vascular care intervention to prevent dementia (preDIVA): a cluster-randomised controlled trial. Lancet. 2016;388(10046):797-805. https://doi.org/10.1016/ s0140-6736(16)30950-3.

32. Andrieu S, Guyonnet S, Coley N, Cantet C, Bonnefoy M, Bordes S, et al. Effect of long-term omega 3 polyunsaturated fatty acid supplementation with or without multidomain intervention on cognitive function in elderly adults with memory complaints (MAPT): a randomised, placebo-controlled trial. The Lancet Neurology. 2017;16(5):377-89. https://doi.org/10.1016/ s1474-4422(17)30040-6.

33. Lamb SE, Sheehan B, Atherton N, Nichols V, Collins H, Mistry D, et al. Dementia And Physical Activity (DAPA) trial of moderate to high intensity exercise training for people with dementia: randomised controlled trial. BMJ. 2018;361:k1675. https://doi.org/10.1136/bmj.k1675.

34. Groot C, Hooghiemstra AM, Raijmakers PG, van Berckel BN, Scheltens P, Scherder $E J$, et al. The effect of physical activity on cognitive function in patients with dementia: a meta-analysis of randomized control trials. Ageing Res Rev. 2016;25:13-23. https://doi.org/10.1016/j.arr.2015.11.005

\section{Publisher's Note}

Springer Nature remains neutral with regard to jurisdictional claims in published maps and institutional affiliations.

Ready to submit your research? Choose BMC and benefit from:

- fast, convenient online submission

- thorough peer review by experienced researchers in your field

- rapid publication on acceptance

- support for research data, including large and complex data types

- gold Open Access which fosters wider collaboration and increased citations

- maximum visibility for your research: over $100 \mathrm{M}$ website views per year

At $\mathrm{BMC}$, research is always in progress.

Learn more biomedcentral.com/submissions 